Review

\title{
Polymer-Based Prodrugs: Improving Tumor Targeting and the Solubility of Small Molecule Drugs in Cancer Therapy
}

\author{
Sonja Dragojevic, Jung Su Ryu and Drazen Raucher* \\ Received: 25 September 2015 ; Accepted: 17 November 2015 ; Published: 4 December 2015 \\ Academic Editors: Thomas Rades, Holger Grohganz and Korbinian Löbmann \\ Department of Biochemistry, University of Mississippi Medical Center, 2500 North State Street Jackson, \\ MS 39216, USA; sdragojevic@umc.edu (S.D.); jryu@umc.edu (J.S.R.) \\ * Correspondence: draucher@umc.edu; Tel.: +1-601-984-1510
}

\begin{abstract}
The majority of anticancer drugs have poor aqueous solubility, produce adverse effects in healthy tissue, and thus impose major limitations on both clinical efficacy and therapeutic safety of cancer chemotherapy. To help circumvent problems associated with solubility, most cancer drugs are now formulated with co-solubilizers. However, these agents often also introduce severe side effects, thereby restricting effective treatment and patient quality of life. A promising approach to addressing problems in anticancer drug solubility and selectivity is their conjugation with polymeric carriers to form polymer-based prodrugs. These polymer-based prodrugs are macromolecular carriers, designed to increase the aqueous solubility of antitumor drugs, can enhance bioavailability. Additionally, polymer-based prodrugs approach exploits unique features of tumor physiology to passively facilitate intratumoral accumulation, and so improve chemodrug pharmacokinetics and pharmacological properties. This review introduces basic concepts of polymer-based prodrugs, provides an overview of currently emerging synthetic, natural, and genetically engineered polymers that now deliver anticancer drugs in preclinical or clinical trials, and highlights their major anticipated applications in anticancer therapies.
\end{abstract}

Keywords: polymer based prodrugs; anticancer drugs; macromolecules; drug delivery; cancer therapy; genetically engineered biopolymers; synthetic and natural polymers

\section{Introduction}

Cancer is one of the major fatal diseases, with current treatments of limited therapeutic efficacy, despite great progress in a range of approaches to tumor eradication, including chemotherapy. The mechanism of action of anti-cancer drugs relies on arresting the cell cycle and rapidly killing all proliferating cells. This killing includes non-cancerous cells, such as bone marrow, gut epithelia, lymphatic system, red blood cells and hair follicles. One of the main challenges in current chemotherapeutic treatments is drug toxicity to healthy organs due to lack of selectivity. To achieve therapeutic efficacy, it is often necessary to administer high doses of drugs because of the drugs' physicochemical properties, such as short circulation time in the blood plasma. The problems associated with the low specificity and poor pharmacokinetics and pharmacodynamics of chemotherapeutic drugs are well known; however, another frequently encountered difficulty is their poor aqueous solubility, which restricts bioavailability.

Intractable problems besetting drug solubility in aqueous solutions have imposed serious clinical constraints on ascertaining effective therapeutic plasma concentrations so as to achieve appropriately targeted pharmacological responses. At present, ensuring the presence of a therapeutic concentration of poorly soluble drugs in systemic circulation requires the administration of high concentrations of potent chemotherapeutics, with their solubility related to particle size. Smaller particles have surface 
area to volume ratio increases, which allows greater interaction with the solvent [1]. The most common methods for reducing particle size are micronization and nano suspension [2]. Micronization relies on mechanical stress, using a technique such as spray drying to break up drug aggregates. Nano suspension relies on forcing the drug suspension under pressure through a nano aperture valve, resulting in drug nanoparticles that can then be stabilized by surfactants. Other new techniques for reducing particle size are also forthcoming, such as sonocrystalization, supercritical fluid processes and wet milling $[3,4]$.

Despite successes within each of these approaches, scientists continue to encounter major concerns related to particle size reduction. These concerns largely arise owing to the strong tendency for particles to agglomerate subsequent to size reduction. Such agglomerates are likely to reduce the drug's therapeutic activity.

A drug's chemical structure and property determine possible approaches for improving their solubility in aqueous solutions. As drugs may be weakly acidic, weakly basic or hydrophobic, appropriate approaches for increasing their rate of solubility may include $\mathrm{pH}$ adjustment, chemical modification, micellar solubilization, hydrotropy, solid dispersion, complexations, microemulsions and co-solvency [5]. Each method possesses advantages, but may also introduce challenges in formulation design, with consequent limitations in therapeutic efficacy. For example, drugs that can be dissolved in water by adjusting their $\mathrm{pH}$ may precipitate in blood upon intravenous administration, due to the strong buffering capacity of blood, with its $\mathrm{pH}$ between 7.2 and 7.4 [6], leading to emboli.

Similarly, another promising approach for improving drug solubility relies upon mixing hydrophobic drugs with co-solutes, which can open more hydrophilic moieties and so facilitate solubility. However, co-solutes often exert more toxic and immunogenic effects, limiting their effective use at doses required for eradicating cancer cells.

One typical example is Paclitaxel, a microtubule stabilizing agent that has been deemed one of the most significant advances in anticancer therapy $[7,8]$. This promising anti-cancer agent, however, has poor water solubility-but it does dissolve in organic solvents. Among the formulations used to increase Paclitaxel solubility for intravenous administration is a vehicle containing a blend of ethanol and Chremophor, or polyethoxyated castor oil. The side effects of Chremophor, however, include neurotoxicity, nephrotoxicity, vasodilatation lethargy, and hypersensitivity reactions mediated by histamine release, requiring premedication with corticosteroids and antihistamines [9]. To reduce these side effects' intensity and so increase Paclitaxel's safety, better strategies for improving its aqueous solubility are urgently needed. Moreover, even were micronization and/or co-solubilization to improve Paclitaxel's solubility, a method for selective delivery of this small molecule to tumor cells is equally important, to prevent its quick distribution to healthy cells in all organs immediately after administration.

Due to the side effects of both anticancer drugs and their co-solutes, a critical need exists for improvements in such drug pharmaceutical properties as solubility, stability, and distribution. Toward this end, many drug delivery systems have been developed, including: (1) lipid based drug delivery systems, such as liposomes, niosomes and solid lipid particles; (2) nanotechnology-based drug delivery systems, including micelles, fullerenes, dendrimers, carbon nanotubes, quantum dots, metal-based nanoparticles, and nanofibers; and (3) polymeric delivery systems, including polymeric micelles, polymeric vesicles, polymer protein or polymer small drug conjugates [10].

At administration, antitumor drugs are quickly distributed in the body, exhibiting fast renal clearance, short durations of action, and considerable dissipation, with only a fraction reaching cancer cells. By conjugating these small molecule agents with a high molecular weight polymer, an increase in their solubility and bioavailability can be attained [11] so as to slow renal elimination, lengthen construct duration in blood circulation and improve the construct's pharmacokinetic profile. A further contribution of such water-soluble macromolecular carriers lies in their ability to facilitate a more targeted drug delivery by exploiting the disorganized vasculature and poor lymphatic drainage that characterizes tumor physiology. This targeting occurs through a phenomenon collectively known as the enhanced permeation and retention effect (EPR) Figure 1. [12]. Exploiting the EPR effect for tumor targeting in this way permits polymer-drug conjugates to be applied at higher doses than 
possible for any free drug, as polymer-bound drugs will preferentially accumulate and release within tumors. With more drug delivered specifically to a tumor, drug toxicity and side effects will be reduced, and overall therapeutic outcomes improved.

Small molecule drug distribution
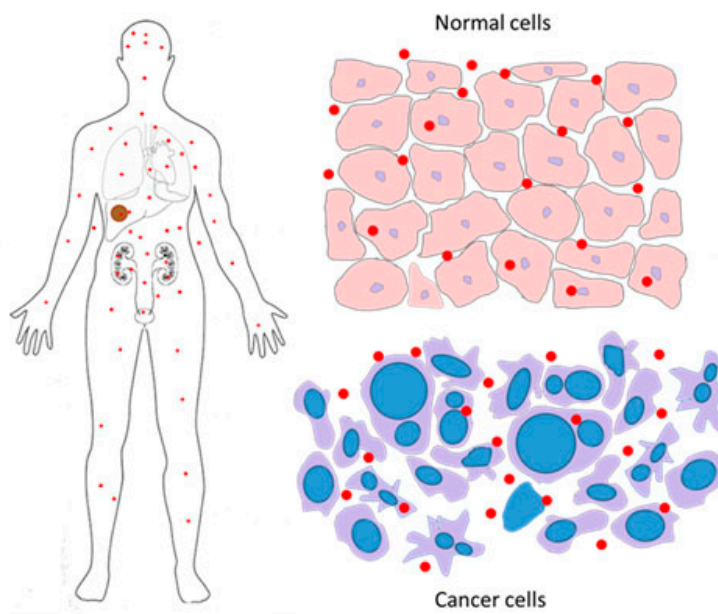

Normal cell

Cancer cell

Tumor tissue
Polymeric based prodrug distribution

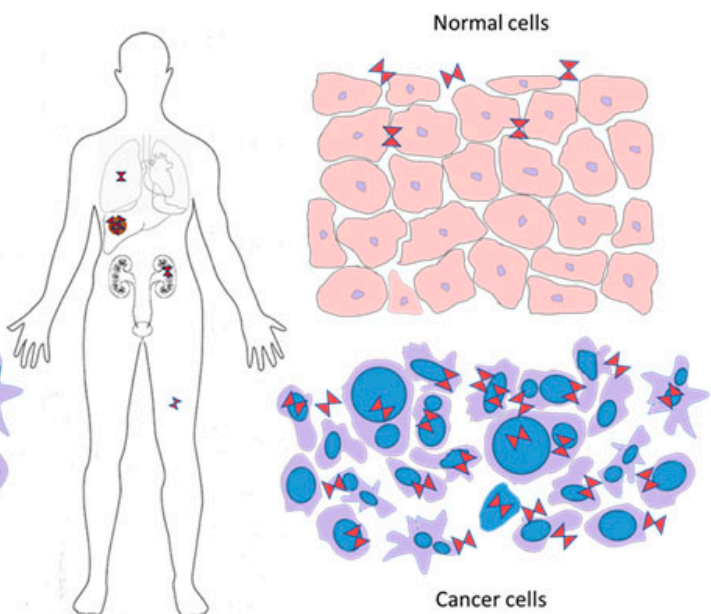

Small molecule drug

Polymer based prodrug

Figure 1. Small molecule drugs are distributed not only in tumor tissue, but are also widely distributed in all healthy tissues, resulting in adverse side effects (left); The polymeric macromolecular carrier is designed to spare healthy tissue from toxicity by preferentially accumulating within tumors, primarily by exploiting their disorganized tumor vasculature and poor lymphatic drainage through the enhanced permeation and retention (EPR) effect (right).

Advances in polymer science have resulted in the synthesis and design of polymers with unique properties. While once confined to roles as co-solutes or mechanical scaffolds, by which to stabilize and solubilize drugs or to control drug release, respectively, polymers now have sophisticated and advanced properties that can be engaged for the development of novel drug delivery vehicles. Prime examples of these advances can be seen in the recently developed synthetic and genetically engineered polymers that can respond to changes in such environmental conditions as $\mathrm{pH}$ or temperature. These new constructs currently underlie active research in the development of innovative approaches for active tumor cell targeting [13-15].

To optimize their potential as drug carriers, polymers must be well characterized, reproducibly synthesized in quantities, and of a purity acceptable for clinical application. They should also contain the appropriate functional groups needed for coupling or incorporation with specific drugs. Not least, they should be composed of biocompatible, non-immunogenic, and biodegradable materials, remain stable in circulation, but readily release their chemodrug cargo intratumorally or intracellularly. Polymer drug carriers should also maintain a low variability in particle size distribution to ensure the homogeneity of polymer-drug conjugates. By their potential to meet these requirements, synthetic, natural and genetically engineered protein polymers can each contribute urgently needed properties to contemporary drug delivery technology.

Synthetic polymers are human-made polymers, usually fabricated with organic solvents such as polyethylene glycol or $\mathrm{N}$-(2-Hydroxypropyl) methacrylamide [16]. Although the structure of synthetic polymers can be easily modified to permit their wide use in drug delivery, problems associated with toxic degradation products or residual toxic components that arise during the process of chemical synthesis have been reported [17]. Natural polymers include polysaccharides, such as 
dextran and chitosan, polypeptides, and polynucleotides. The use of these polymers as drug-delivery agents is widely reported in cell culture and preclinical trials; however, they have not yet reached clinical trials despite offering greater biocompatibility and biodegradability than synthetic polymers. Genetically engineered polypeptides, developed for controlled and targeted drug delivery, offer polymers with uniform composition and precise molecular weight as compared with chemically synthesized products. These constructs are currently under further development within preclinical trials [15].

In summary, cancer therapies that rely on the systemic administration of anticancer agents face considerable obstacles, with poor solubility main among them. This insolubility, through the consequently lowered bioavailability of chemotherapeutic agents, has to date frustrated treatment efforts and led to the taxing outcome of moderate cancer treatment efficacy. The coupling of antitumor drugs with polymeric drug carriers, whether synthetic, natural or genetically engineered, can help overcome agent insolubility, rendering cancer treatment more effective. These polymers can potentially provide vehicles through which to improve application and target delivery of small, poorly water-soluble chemodrugs so as to improve therapeutic effectiveness and reduce the toll of treatment for cancer patients.

\section{Classification of Polymers}

\subsection{Synthetic Polymers}

The advancement of modern polymer chemistry has allowed the modification of polymer structures, rendering "a tailored polymer for the need" possible. For this reason, a wide variety of synthetic polymers, characterized by variations in the main chain as well as in side chains, are currently available (Table 1).

\subsubsection{Polyethylene Glycol (PEG)}

One of the most well-known synthetic polymers, polyethylene glycol (PEG), is a linear polyether compound with ethylene glycol repeats. PEG terminates with hydroxyl group, and can readily be covalently conjugated to many peptides, proteins or drugs (Figure 2). For the coupling of PEG and drugs, agents such as dicyclohexyl carbodiimide (DCC), 1-ethyl-3-(3-dimethylaminopropyl) carbodiimide (EDC), or N-hydroxysuccinimide (NHS) esters are commonly used.

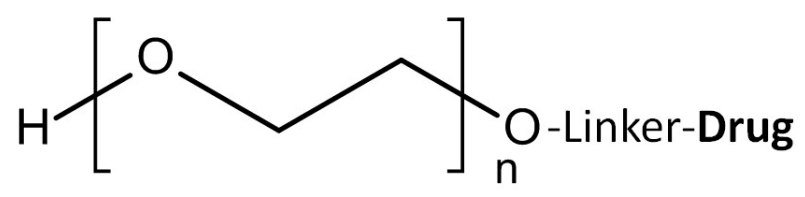

Figure 2. Structure of polyethylene glycol (PEG) and its drug conjugation.

The incorporation of PEG with a drug has the advantage of increasing solubility and therefore bioavailability of the resulting conjugates. Additionally, immunogenic responses upon exposure to peptides/proteins may be diminished by the presence of PEG, which can prevent exposure of the peptide's epitopes and thereby mask peptide/protein recognition by the reticuloendothelial system (RES) $[18,19]$. The main problem with using PEG, however, lies in its poor drug-loading efficiency, with the limited number of reactive groups in PEG often restricting its broad applications as a drug carrier [20]. 
Table 1. Recent applications of synthetic polymers in prodrug delivery.

\begin{tabular}{|c|c|c|c|c|c|}
\hline Polymer & Application & (co)Polymer & Prodrug & Linker(bond) & Ref \\
\hline \multirow{16}{*}{ PEG } & Gene delivery & Polyethylenimine(PEI)-PEG & $\begin{array}{l}\text { cDNA of herpes simplex virus thymidine kinase } \\
\text { gene (HSVtk) and granulocyte-macrophage } \\
\text { colony-stimulating factor (GM-CSF) }\end{array}$ & N.D. & {$[21]$} \\
\hline & Tumor targeting & octreotide(Phe)-PEG & Paclitaxel & & {$[22]$} \\
\hline & $\begin{array}{l}\text { Improved stability and } \\
\text { intracellular drug release }\end{array}$ & $\begin{array}{l}\text { methoxy PEG- } b \text {-(poly(2-(diisopropylamino) } \\
\text { ethyl methacrylate-co-aminopropyl } \\
\text { methacrylamide) (PEDPA) }\end{array}$ & Cis-aconityl-doxorubicin & & {$[23]$} \\
\hline & Tumor targeting & PEG & $\begin{array}{l}\text { Fusion toxin consisting of the anti-EpCAM DARPin } \\
\text { Ec1 and a domain I-deleted variant of ETA (ETA) }\end{array}$ & $\begin{array}{l}\text { rhinovirus } 3 \mathrm{C} \text { model } \\
\text { protease-cleavage linker }\end{array}$ & {$[24]$} \\
\hline & Drug delivery & $\begin{array}{l}\text { 3,3'-dithiodipropionic acid functionalized } \\
\text { PEG- } b \text {-poly(l-lysine) (mPEG- } b \text {-P(LL-DTPA)) }\end{array}$ & Paclitaxel & Disulfide & {$[25]$} \\
\hline & Multidrug resistance & D- $\alpha$-tocopherol PEG succinate (TPGS) & Paclitaxel & Disulfide & {$[26]$} \\
\hline & $\begin{array}{l}\text { Improved the } \\
\text { therapeutic efficacy }\end{array}$ & $\beta-\mathrm{CD}, \mathrm{PEG}$ & Doxorubicin & hydrazone & {$[27]$} \\
\hline & Tumor targeting & PEG & Paclitaxel & & {$[28]$} \\
\hline & Multidrug resistance & PEG-poly(D,L-lactide) & 4-(N)-stearoyl Gemcitabine & & [29] \\
\hline & Theranosis & PEG-polylactic acid (PEG-PLA) 1 & Dicyanomethylene-4H-pyran-S-CPT & Disulfide & [30] \\
\hline & Tumor targeting & PEG monomethyl ether (mPEG) & Artesunate & Ester & [31] \\
\hline & Tumor targeting & PEG monomethyl ether & Camptothecin & Disulfide & [32] \\
\hline & Tumor targeting & PEG & Camptothecin & Disulfide & {$[33]$} \\
\hline & Tumor targeting & PEG2000 & Paclitaxel & MMP2-cleavable linker & {$[34]$} \\
\hline & Nanogel & PLGA-PEG-PLGA & PEGylated Taxol & & {$[35]$} \\
\hline & Drug delivery & mPEG-b-P(ATMC-co-DTC) & Doxorubicin & Hydrazone & {$[36]$} \\
\hline
\end{tabular}


Table 1. Cont.

\begin{tabular}{|c|c|c|c|c|c|}
\hline Polymer & Application & (co)Polymer & Prodrug & Linker(bond) & Ref. \\
\hline \multirow{14}{*}{ HPMA } & $\begin{array}{l}\text { improving } \\
\text { anticancer therapy }\end{array}$ & (mPEG5000-b-p(HPMAmLac2- $r$-AzEMA) & $\begin{array}{l}\text { Doxorubicin-glucuronide prodrug } \\
\text { (DOX-propGA3) }\end{array}$ & $\begin{array}{l}\text { Glucuronide } \\
\text { ( } \beta \text {-glucuronidase cleavable linker) }\end{array}$ & [37] \\
\hline & Prevent metastasis & HPMA copolymer & $\begin{array}{l}\text { E-selectin binding peptide (Esbp)-doxorubicinor } \\
(\mathrm{KLAKLAK})_{2}\end{array}$ & & [38] \\
\hline & Drug delivery & HPMA copolymer & H1-S6A, F8A peptide & GFLG (Cathepsin cleavage linker) & [39] \\
\hline & Tumor targeting & HPMA copolymer & Doxorubicin & GFLG and MMP cleavable linker & [40] \\
\hline & $\begin{array}{l}\text { Improved the } \\
\text { therapeutic efficacy }\end{array}$ & HPMA copolymer & Doxorubicin, 5-FU & Hydrazone, GFLG & [41] \\
\hline & Theranosis & $\begin{array}{l}\text { Star polymer: poly(amido amine) (PAMAM) } \\
\text { dendrimers and HPMA }\end{array}$ & Doxorubicin or TAMRA fluorescent dye & Hydrazone & [42] \\
\hline & Improved Bioavailability & $\begin{array}{l}\text { Star polymer: poly(amido amine) (PAMAM) } \\
\text { dendrimers and HPMA }\end{array}$ & Pirarubicin & Hydrazone & {$[43,44]$} \\
\hline & Drug delivery & HPMA copolymer & Iodine-125 & Hydrazone & [45] \\
\hline & Drug delivery & HPMA copolymer & Paclitaxel, Gemcitabine & GFLG & [46] \\
\hline & Drug delivery & Starch + HPMA copolymer & Camptothecin & & [47] \\
\hline & Gene delivery & $\begin{array}{l}\text { galactosylated 2-hydroxypropylmethacrylamide- } \\
s \text {-3-guanidinopropyl methacrylamide } \\
\text { (HPMAs-GPMA) }\end{array}$ & shRNA & & [48] \\
\hline & Drug delivery & HPMA & Indium-111, Yttrium-90 & & [49] \\
\hline & $\begin{array}{l}\text { Improved the } \\
\text { therapeutic efficacy }\end{array}$ & multiblock poly HPMA & Gemcitabine, Paclitaxel and Doxorubicin & GFLG & [50] \\
\hline & Theranosis & HPMA copolymer & Zinc protoporphyrin & & {$[16,51]$} \\
\hline SMA & Photodynamic therapy & SMA & Zinc protoporphyrin & Amide & {$[52]$} \\
\hline PLGA & Improved Bioavailability & PLGA & Gemcitabine & Amide & [53] \\
\hline PGG & Improved Bioavailability & PGG & Paclitaxel & Glutamic acid & {$[54,55]$} \\
\hline
\end{tabular}


In spite of these limitations, PEG has been used to deliver a variety of chemical, peptide and gene therapies. For example, Chen et al., produced a Paclitaxel prodrug conjugated to poly (ethylene glycol)- $b$-poly (l-lysine) by connecting the drug to the polymer via a disulfide bond. This allowed the Paclitaxel to be released from the polymer conjugates when reduced by glutathione, widely utilized as the ideal trigger in redox-responsive delivery systems [25]. Other examples are found in the study of Cai et al. [56]. They modified PEG with functional dendrimers to fabricate linear-dendritic copolymers with multiple reactive groups, increasing the number of reactive groups in PEG-based polymers and improving the treatment efficacy of the conjugated drugs [56]. Xia et al. also developed a strategy conjugating PEG with multi-numbers of drugs, such as paclitaxel and cisplatin, to maximize the treatment efficiency of the drug-polymer conjugates [57].

\subsubsection{The N-(2-hydroxypropyl) Methacrylamide (HPMA) Copolymer}

After early uses of a HPMA monomer/homopolymer as a plasma expander, HPMA's conjugation with doxorubicin in 1994 clinical trials [58] to its extensive application and investigation for cancer treatment, especially as a copolymer. HPMA, like PEG, is a water soluble, biocompatible, non-immunogenic polymer. Unlike PEG, however, the large number of pendent functional groups in HPMA copolymers permits the conjugation of many drugs (Figure 3). Further, HPMA facilitates activating or releasing the compound from the polymer backbone within a targeted region by manipulation of their bond, a degradable linker. For example, Huang et al. used a GFLG cathepsin cleavable linker to deliver a c-Myc inhibitory peptide, the H1-S6A, F8A peptide. An increase in nuclear drug accumulation and an inhibition of tumor growth were achieved with a construct composed of a nuclear localization sequence (NLS) and the polymer complex (R8NLS-HPMAcoPolymer-GFLGH1-S6A, F8A) [39]

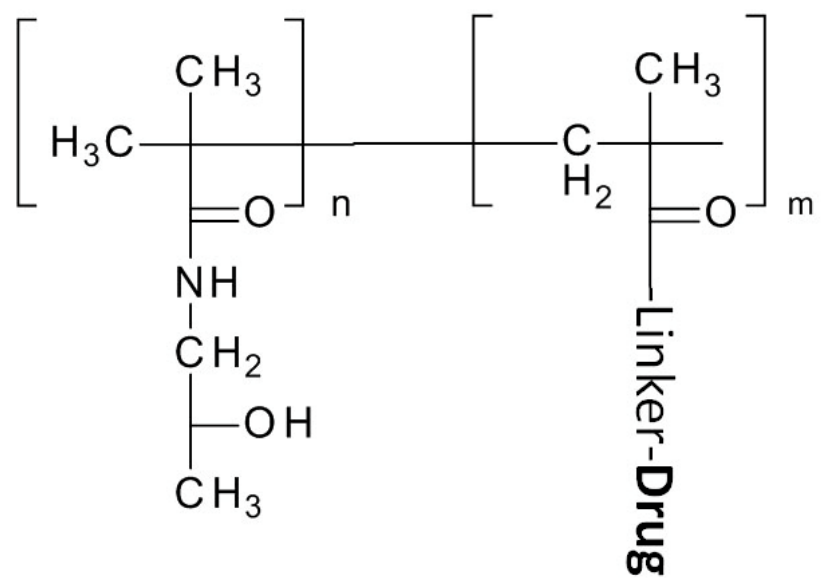

Figure 3. Structure of N-(2-hydroxypropyl) Methacrylamide (HPMA) and its drug conjugation.

In another study, Etrych et al. applied an acid-sensitive hydrazone linker to bridge the HPMA-poly (amide amine) (PAMAM) dendrimers copolymer [42]. In this study, a fluorescent dye, TAMRA, was also conjugated to this dendrimer to visualize the feasibility of the polymer carrier by in vitro optical imaging.

\subsubsection{Poly (Styrene-Co-Maleic Acid/Anhydride) (SMA)}

Poly (styrene-co-maleic acid/anhydride) (SMA) is an alternating copolymer composed of styrene and maleic anhydride (Figure 4). Since SMA forms micelles with a hydrophobic styrenic core and a hydrophilic maleic acid surface, a stable and rate-controllable release of micelles can allow surface modification for use in tissue targeting [59]. Although research on SMA is not as active as that for PEG or HPMA, studies have continued to aim at producing prodrugs using this polymer. In 1985, Maeda et al. developed a conjugate between SMA and neocarzinostatin (SMANCS) by using an 
amide bond between the peptide's terminal amino group and the SMA carboxylanhydride [60]. This work yielded a decreased clearance rate and an increase in the tumor concentration of neocarzinostatin. The enhanced bioavailability and tumor accumulation achieved by this conjugate led to the effective treatment of a variety of solid tumors [61,62]. Recently, Maeda et al. developed a styrene-maleic acid-copolymer conjugated with ZnPP (SMA-ZnPP) for photodynamic therapy [63]. In addition to increased tumor accumulation achieved through the EPR effect, light irradiation increased the complex's cytotoxicity up to six fold.
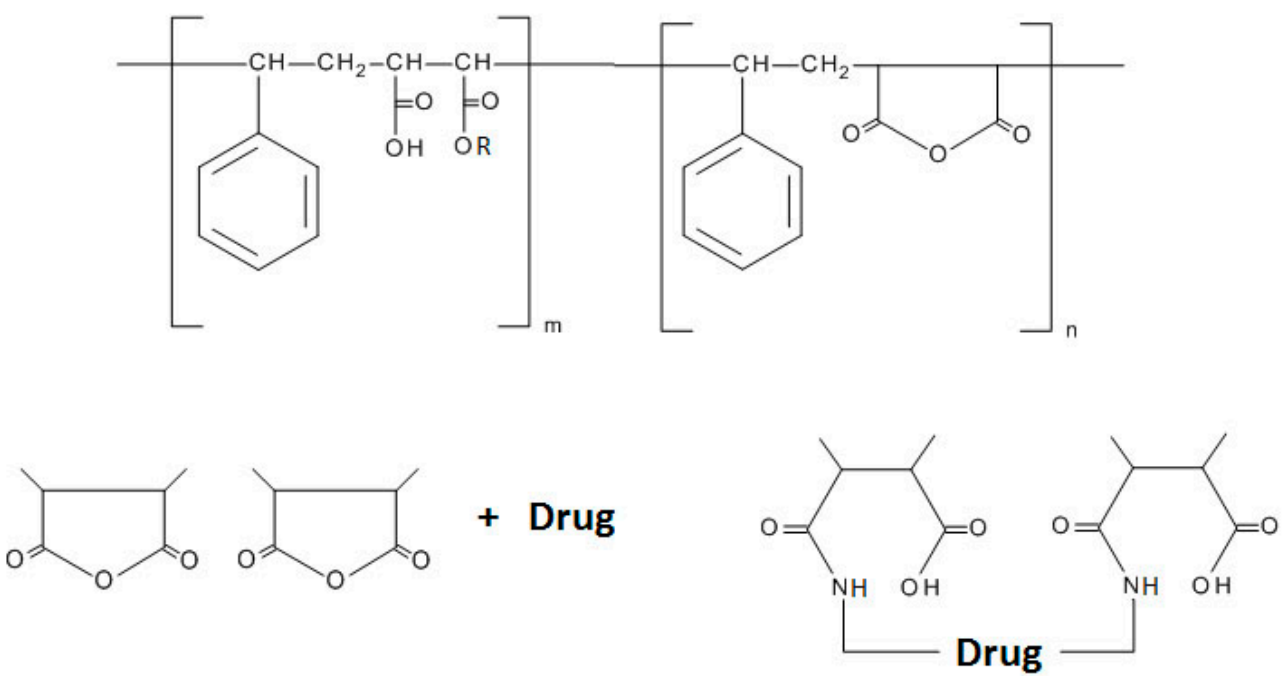

Figure 4. Structure of Poly (styrene-co-maleic acid/anhydride) (SMA) and its drug conjugation [61].

\subsubsection{The Polyglutamic Acid Polymer}

Bae et al. [64] developed a polymer nanogel based on poly ( $\gamma$-glutamic acid) ( $\gamma$-PGA) (Figure 5). Poly ( $\gamma$-glutamic acid) ( $\gamma$-PGA), a highly anionic polymer which exhibits excellent biocompatibility and non-cytotoxicity, is naturally synthesized in microbial species, especially bacilli. Bae and colleagues developed a thiolated $\gamma$-PGA nanogel for doxorubicin delivery that, when used to treat MCF 7 breast cancer cells, showed controlled drug release behavior and higher toxicity as compared to free doxorubicin. Their approach suggests that thiolated $\gamma$-PGA nanogel may be a promising drug delivery vehicle in anticancer therapy [64]. Furthermore, in the study of Yu et al. [54], the PGA was added with another glutamic acid to each glutamic acid in the polymer backbone producing a poly(L- $\gamma$-glutamyl-glutamine)(PGG). This modification shows additional hydrophilicity on a PGG-paclitaxel conjugate as well as improved paclitaxel-loading efficiency in comparison to PGA-paclitaxel conjugates.

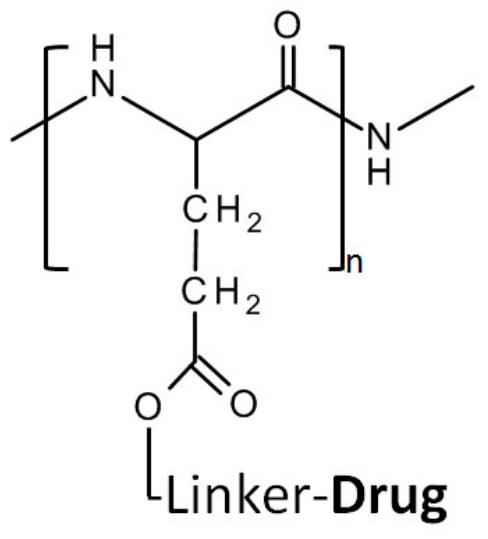

Figure 5. Structure of PGA and its drug conjugation. 
Seth et al., [65]used poly ( $\gamma$-glutamic acid) ( $\gamma$-PGA) in a mouse melanoma tumor model to deliver both the immuno-stimulating agent, toll-like receptor-7 (TLR-7) agonist-imiquimod and the chemotherapeutic agent paclitaxel. These water insoluble therapeutic agents formed crystalline microstructures that were dispersed in the IgPGA matrix. When the complex was administered by intra-tumoral injection, this combined treatment caused synergistic tumor regression as compared with effects observed when each of the compounds was used alone. This study demonstrates an excellent example and practical approach for the use of water soluble polymers, such as poly ( $\gamma$-glutamic acid), in formulating micro-dispersions and delivering combinations of water insoluble drugs [65].

\subsubsection{Poly (Lactic-Co-Glycolic Acid) (PLGA)}

PLGA is a FDA approved biodegradable and biocompatible copolymer of poly lactic acid (PLA) and poly glycolic acid (Figure 6) [66]. This copolymer has been conjugated to gemcitabine via amide bonding [53]. Through the biodegradability of PLGA, the conjugated gemcitabine and released from the complex slowly and over time. Stability and in vitro efficacy testing have shown that PLGA-gemcitabine complex has advantages over free Gemcitabine.

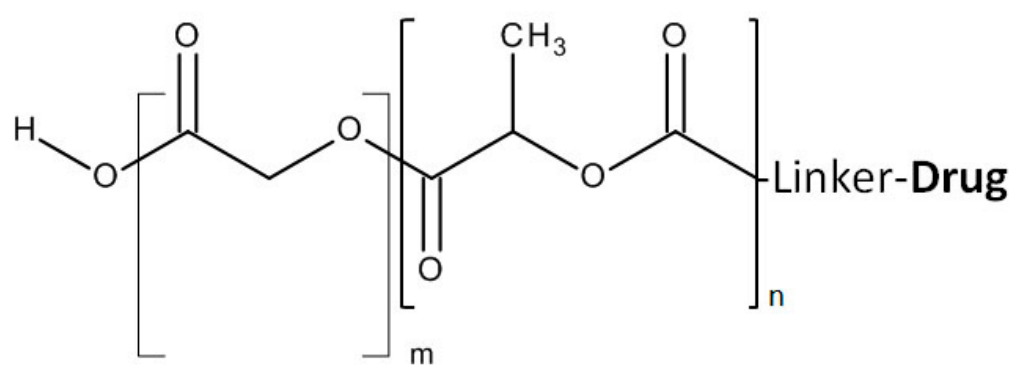

Figure 6. Structure of PLGA and its drug conjugation.

To further improve their drug delivery properties, synthetic polymers have often been combined with natural polymers. The synthetic polymer polyethylene glycol (PEG) and the natural polymer chitosan have been applied as conjugates in both in vitro and in vivo studies; however, the secondary stability and short half-life of chitosan-based micelles has hampered outcomes under in vivo conditions.

Emami et al. [67] used chitosan (see Natural Polymers, below) to develop a novel polymeric micelle with which to deliver paclitaxel. The new micelle, a tocopherol succinate-chitosan-polyethylene glycolfolic acid (TS-CS-PEG-FA), loaded with paclitaxel, yielded both an increased PTX accumulation in tumor tissue and increased toxicity to cancer cells as compared to free PTX. The increased tumor accumulation and favorable pharmacokinetics and tissue distribution observed in this study shows that chitosan-derived micelles have the potential to serve as an effective drug delivery vehicle for paclitaxel [67].

\subsection{Natural Polymers}

Natural polymers are commonly classified in three major classes: polysaccharides, polypeptides and polynucleotides. As polynucleotides are not used as drug carriers in anticancer therapy, only polysaccharides and polypeptides will be discussed below. For a discussion of polynucleotides in cancer therapy, however, interested readers are pointed to the work of Cheng et al. [68], Gorska et al. [69], and Xiang et al. [70].

Polysaccharides and polypeptides can be successfully conjugated with most anti-tumor drugs. Moreover, some of these polymers themselves possess antitumor characteristics. For example, chitosan, through such physical and chemical properties as small particle size and positive surface charge, exerts antitumor activity by disrupting the tumor cell membrane and thereby inducing apoptosis. The results of in vitro and in vivo studies that used chitosan as an anticancer agent against 
sarcomas and hepatocarinomas have furthered the view that chitosan can provide a promising antitumor agent, advancing its progress toward clinical trials [71,72].

Natural polymers can now be exploited as a foundation to which poorly soluble drugs can be attached and delivered to tumor sites. Through their considerable versatility the use of these polymers can occasion less drug loss and lower toxicity to healthy tissue.

\subsubsection{Chitosan}

Chitosan, a linear polymer composed of interchangeably organized, deacetylated $\beta$-(1-4)-linked D glucosamine units and of acetylated $N$-acetyl-D-glucosamine units (Figure 7), is widely used in drug delivery systems. The sole polysaccharide polycation used as a polymer, chitosan binds to negatively charged moieties[20]. Recently, Nogueira and colleagues produced chitosan nanoparticles loaded with the anticancer agent Methotrexate (MTX) [73]. This poorly soluble anticancer agent is extremely potent, but poses serious risks of adverse effects to normal cells, including kidney failure, neurotoxicity and mucositis [73-75].

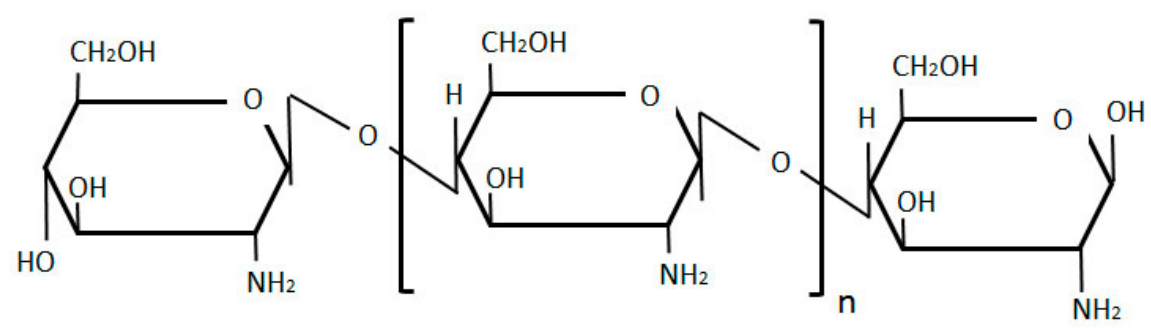

Figure 7. Chitosan structure, composed of deacetylated $\beta-(1-4)$-linked D-glucosamine units and of acetylated $N$-acetyl-D-glucosamine units.

To overcome chitosan's toxicity and poor solubility, Nogueira et al. [73] constructed chitosan-based nanoparticles encapsulating methotrexate (MTX-CS-NPs). Next, to make the chitosan-based nanoparticles $\mathrm{pH}$ responsive, these researchers added the amino acid based amphiphile surfactant, 77KS. The incorporation of this $\mathrm{pH}$-sensitive moiety permitted a $\mathrm{pH}$-dependent, controlled release of Methotrexate. In vitro experiments were conducted in MCF7 breast cancer cells, with promising results. Future in vivo studies will provide more information about the clinical potential of chitosan-based nanoparticles.

Another promising approach using grafted-chitosan polymeric micelles carrying two drugs was developed by Nam et al. [76]. The chitosan derivative O-carboxymethyl chitosan (OCMCh), with enhanced solubility, was conjugated first with $\alpha$-tocopherol, forming $\alpha$-tocopherol $O$-carboxymethyl (TOC). This chitosan polymer was then ligated with doxorubicin and an anti-human epidermal growth factor receptor 2 [HER2] target peptide, producing the final construct, HPTOC-DOX polymeric micelles. An in vitro study that employed this construct against the SK-BR-3 cell line showed a synergistic effect of tocopherol and dox. Furthermore, in an in vivo study with SK-BR-3 tumor bearing mice, the anti-HER targeting peptide enhanced not only cellular uptake, but also therapeutic efficacy.

The advantages of chitosan include that it is biodegradable and biocompatible, as well as able to efficiently transport polar drugs across an epithelial surface. In addition, chitosan oligomer derivatives of 3-6 kDa are considered relatively nontoxic. Chitosan's major disadvantage is its limited solubility at physiological $\mathrm{pH}$, although this can be overcome by combining it with other polymers and/or by chemical modification.

\subsubsection{Dextran}

Dextran, structured of glucose molecules that form a complex branched glucan chain of variable length, is a water soluble polymeric saccharide (Figure 8). Dextran-based microspheres have been explored in in vitro, in vivo and most recently, in clinical trials of breast, colon, hepatic, 
and pancreatic tumors (please see Table 2. below). These microspheres can satisfy the vast majority of drug carrier requirements. Their primary use in cancer research is to improve the solubility of insoluble anti-tumor drugs.

The slow release systems associated with microspheres have made dextran microspheres (MS) the vehicle of choice for the delivery of mitomycin $\mathrm{C}(\mathrm{MMC})$, a promising, potent anticancer agent that functions through bioreductive activation [77]. Many acute as well as chronic toxicities are associated with MMC, however, severely limiting its clinical application. To overcome these impediments and achieve targeted drug delivery to the hypoxic regions of solid tumors, Cheung and colleagues developed a targeted approach composed of oxidized dextran microspheres loaded with MMC [77]. In an in vitro model of breast cancer cell-line, EMT6, these researchers applied a doxorubicin-MS therapy, followed by a MMC-MS therapy. This sequential application of two antitumor drugs resulted in a synergistically effective combination of cancer cell killing.

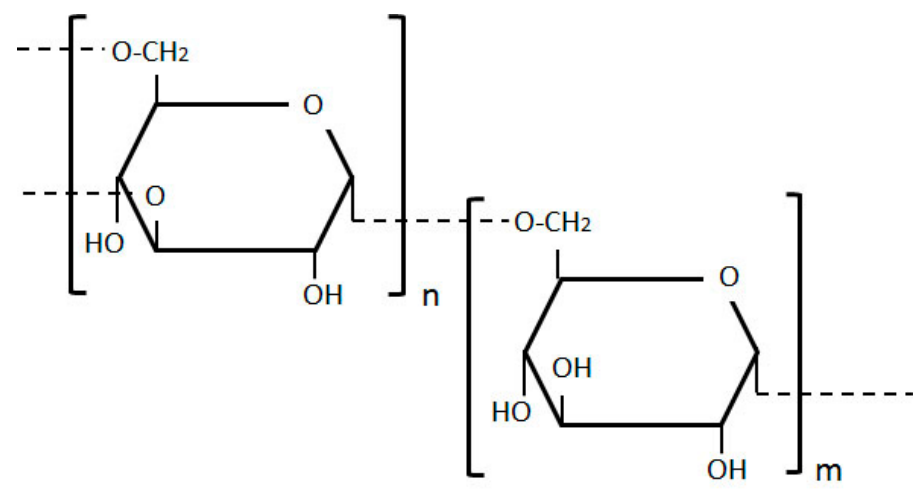

Figure 8. Dextran structure, composed of glucose molecules forming a complex branched glucan chain.

Table 2. Current clinical trials of investigational anticancer drugs based on polymer.

\begin{tabular}{ccccc}
\hline Polymer & Name & Drug & Status & Ref. \\
\hline \multirow{2}{*}{ Dextran } & OsteoDex & Alendronate & Phase I & {$[21,78,79]$} \\
& Somadex & Somatostatin & Phase I & {$[78]$} \\
\hline \multirow{2}{*}{ PEG } & NK105 & Paclitaxel & Phase III & {$[21]$} \\
& NK102 & SN-38 & Phase II & {$[80,81]$} \\
& NC-6004 & Cisplatin & Phase III & {$[21]$} \\
& NC-4016 & Dachplatin & Phase I & {$[21]$} \\
& NC-6300 & Doxorubicin & Phase I & {$[21]$} \\
\hline poly-L-glutamate & paclitaxel poliglumex, CT-2103 & Paclitaxel & Phase III & {$[82,83]$} \\
\hline Cyclodextrin-PEG copolymer & CRLX101 & Camptothecin & Phase II & {$[84]$} \\
\hline
\end{tabular}

Dextran microspheres exhibit biodegradability and biocompatibility, as well as being nonimmunogenic and nontoxic, all important factors for future clinical applications. These microspheres, easily filtered and neutral, are above all water soluble. However, their clinical translation has been severely hampered by the vexing problem that, when conjugated to antitumor drugs, dextran is rendered immunogenic and non-biodegradable [20]. Other negative effects of dextran polymer use currently include platelet dysfunction, anaphylaxis, and pulmonary, as well as cerebral edema. Studies to determine whether or not these problems can be successfully resolved are now needed.

For nanoparticle assembly, it is important that an anticancer agent/drug and polymer have opposite ionic properties. Therefore, the incorporation of ionic macromolecules with oppositely charged ionic-molecules to form stable nanoparticles can be beneficial [85]. In order to combine advantages and circumvent disadvantages, Lee et al. [85] conjugated chitosan and dextran into self-organized nanoparticles for doxorubicin tumor targeted delivery. 


\subsubsection{Pullulan}

Pullulan, a natural polysaccharide composed of maltotriose units structured as $\alpha-1-4 ; \alpha-1-6$-glucan (Figure 9.) and a liver specific biopolymer [86], is biodegradable, nontoxic, non-mutagenic, and noncarcinogenic. Its low immunogenicity and satisfactory solubility in aqueous and several organic solvents have together led to a range of applications in cancer drug delivery.

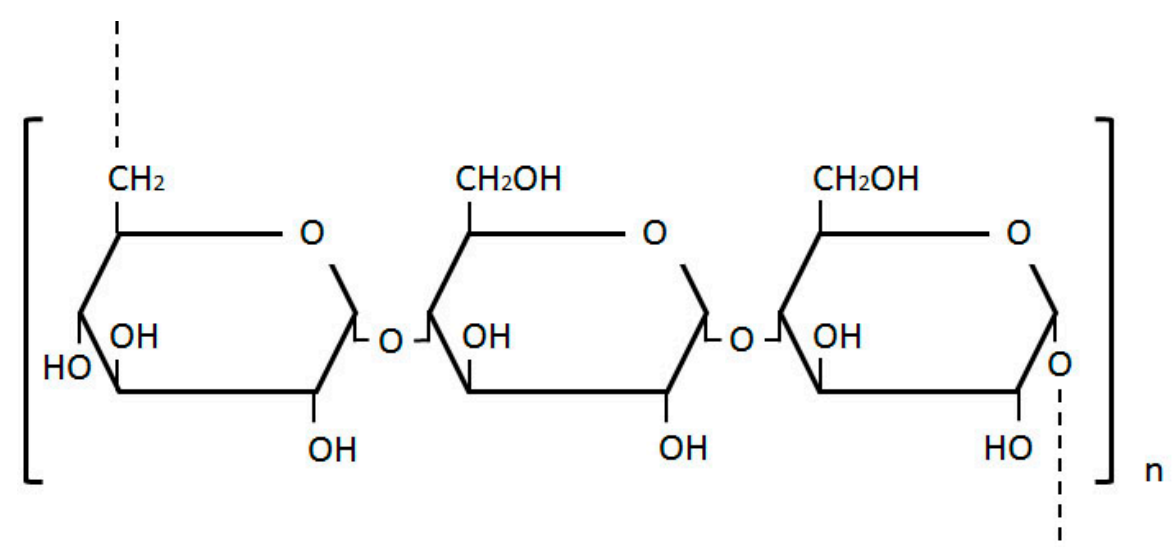

Figure 9. Pullulan structure, composed of maltotriose units.

Scomparin et al. [87] conducted an in vitro study with two pullulan bioconjugates. The aim of their study was to design an anticancer polymer therapeutic for targeted tumor cell therapy. The group synthesized the two pullulan derivatives by conjugating pullalan with (1) doxorubicin and (2) doxorubicin and folic acid, with the latter used as a moiety for targeting folic receptors overexpressed in some tumor cell lines. To achieve proper functionality, the pullulan was activated by such further chemical modifications as periodate oxidation and reductive conjugation with cysteamide. Both derivatives, the folic acid-free derivative [( $\left.\left.\mathrm{NH}_{2} \mathrm{PEG}\right)-\mathrm{Pull}-(\mathrm{cyst}-\mathrm{Dox})\right]$ and the folic acid-doxorubicin-coupled derivative [(FA-PEG)-Pull-(Cyst-Dox)], contained a similar doxorubicin concentration $(w / w)$, i.e., $\sim 6 \%$, with $4.3 \%(w / w)$ folic acid in (FA-PEG)-Pull-(Cyst-Dox). This group's in vitro study results showed reduced toxicity in the folic acid non-expressing receptor cell line, MCF7, but increased toxicity in the KB cell line, which has over-expressed folic acid receptors. This finding demonstrated the specificity of pullulan polymer bioconjugates and the improved pharmacokinetics of doxorubicin. Moreover, Scomparin and colleague's design for actively targeting tumors in folic acid over-expressing receptor tumor cell lines, offering a new approach for in vivo studies of this bioconjugate pullulan.

The synthesis of pullulan-stabilized gold nanoparticles (PAuNPs) was reported by Ganeshkumar et al. [86] for its use in delivering the anticancer drug 5-fluorouracil (5Fu) and folic acid. As described previously, folic acid can be used for active targeting of folate receptor over-expressing cell lines. 5-Fluorouracil was introduced in clinics in 1957 as a poorly-soluble, but potent drug for treating solid tumors. Despite its promise as a chemotherapeutic agent, it showed limited response rates of only 10\%-30\% [88]. Ganeshkumar and colleagues [86] designed their 2014 study in an effort to increase solubility, stability and specificity, as well as to minimize the side effects of this potent agent. In vitro cytotoxicity tests were conducted in free 5-Fu and 5-Fu@AuNPs, as well as in 5-Fu@AuNPs against HepG2 cells with over-expressed folate receptors. Biodistrubution studies in male Wistar rats showed no excessive toxicity in untargeted healthy cells. This group's encouraging results with 5-Fu@PAuNPs-Fa bioconjugates have set the groundwork for a novel approach in active liver cancer targeting.

Using a similar approach, Zhang et al. [89] constructed maleilated pullulan-doxorubicin conjugated with a folate polymeric prodrug for active tumor-targeted delivery, reported as FA-MP-DOX. The purpose of conjugating the construct with folates is to enhance cellular uptake by folate receptor mediated endocytosis. The folate-decorated maleilated pullulan-doxorubicin construct, used to treat ovarian carcinoma A2780 cells, was compared to free doxorubicin and to a maleilated pullulan 
doxorubicin construct. FA-MP-DOX showed higher toxicity in tumor cells, along with enhanced cellular uptake in ovarian carcinoma A278 cells as compared to free doxorubicin and the MP-DOX construct. These findings suggest that the FA-MP-DOX approach may have potential for clinical application in ovarian carcinoma treatment.

\section{Genetically Engineered Polypeptides}

Genetically engineered polypeptides have been gaining recognition in biomaterials and drug delivery fields for decades owing to custom design and simple, inexpensive manufacturing. These polypeptides are genetically encoded, providing control over their sequence and molecular weight (MW) to an extent impossible with synthetic polymer analogs. Moreover, these biopolymers are simple and inexpensive to manufacture as they are expressed in E. coli at high yields and can be easily purified; their custom design further provides opportunities to confer low immunotoxicity and immunogenicity. These polymers are thus now widely used in anticancer drug delivery to improve the solubility of anti-cancer therapeutics.

\subsubsection{Elastin-Like Polypeptide (ELP)}

An ELP is a thermally responsive pentapeptide (VPGXaaG)n amino acid repeat, where Xaa is any guest residue amino acid other than proline; the ELP polymer is easily tailored for a wide variety of applications. Depending on an ELP's primary structure (e.g., amino acid composition), it can form aggregates, hydrogels or micelles in response to stimuli (thermal or $\mathrm{pH}$ ). As an ELP is a macromolecule, it can exploit the EPR effect to achieve passive targeting to solid tumors. Moreover, an ELP is designed to undergo an inverse transition in response to thermal stimuli. Below the transition temperature, the ELP is disordered and soluble in aqueous solution. At an increase in the solution's temperature above the transition temperature through application of a mild, external heat, the ELP forms aggregates and can be actively targeted to tumor tissue.

The ELP has now been extensively applied in targeted anticancer drug delivery [90], an area of research that has now expanded to include breast, prostate, ovarian, and brain tumor studies [91-94]. It has also been used in drug delivery for therapeutic angiogenesis in pre-eclampsia [95] and in biomaterials research [96].

For example, Bidwell et al. [97] conjugated the anticancer drug, doxorubicin, to an ELP composed of Tat, a cell penetrating peptide (CPP), ELP and GFLG, a tetrapeptide linker that is a substrate for the lysosomal cathepsin proteases and doxorubicin (Figure 10.). This therapeutic polymeric prodrug was used to treat sensitive MES/SA cells and resistant MES-SA/Dx5 cells. The study's findings showed that doxorubicin was equally cytotoxic in both cell lines and that ELP-bound doxorubicin accumulated in MES-SA/Dx5 cells, overcoming their drug resistance.

A similar approach was used by Moktan et al. [90] to deliver paclitaxel. This polymeric prodrug was composed of three domains: CPP, ELP and paclitaxel. The paclitaxel prodrug contained hydrazine, an acid sensitive linker that is cleaved at lysosomal pH. The study showed that ELP-delivered paclitaxel combined with mild hyperthermia effectively inhibits proliferation in MCF7 and in multidrug resistant MCF7 cells, providing in vitro proof of concept for the use of ELP-paclitaxel.

Furthermore, in vivo studies support that using ELP as a macromolecular carrier for the delivery of doxorubicin and paclitaxel is a promising approach for clinical application. Moktan et al. [92] conjugated an ELP with a CPP (SynB1) at its N-terminus and a 6-maleimidocaproyl hydrazine derivate of doxorubicin at its C-terminus to compare the efficacy of the construct with that of free doxorubicin. Results showed complete tumor inhibition with SynB1-ELP-Dox, as opposed to only moderate tumor inhibition with free dox. Furthermore, the delivery of dox using the ELP construct minimized the cardiac toxicity commonly present with the free form of doxorubicin.

MacKay developed a different approach to delivering Doxorubicin using ELP forming micelles. The ELP polypeptides were modified with short, cysteine-rich segments that were constructed so as to spontaneously self-assemble into $100 \mathrm{~nm}$ nanoparticles upon conjugation with doxorubicin. The conjugated doxorubicin nanoparticles exhibited a lower systemic toxicity, but induced almost complete tumor regression in a mouse cancer model [98]. 


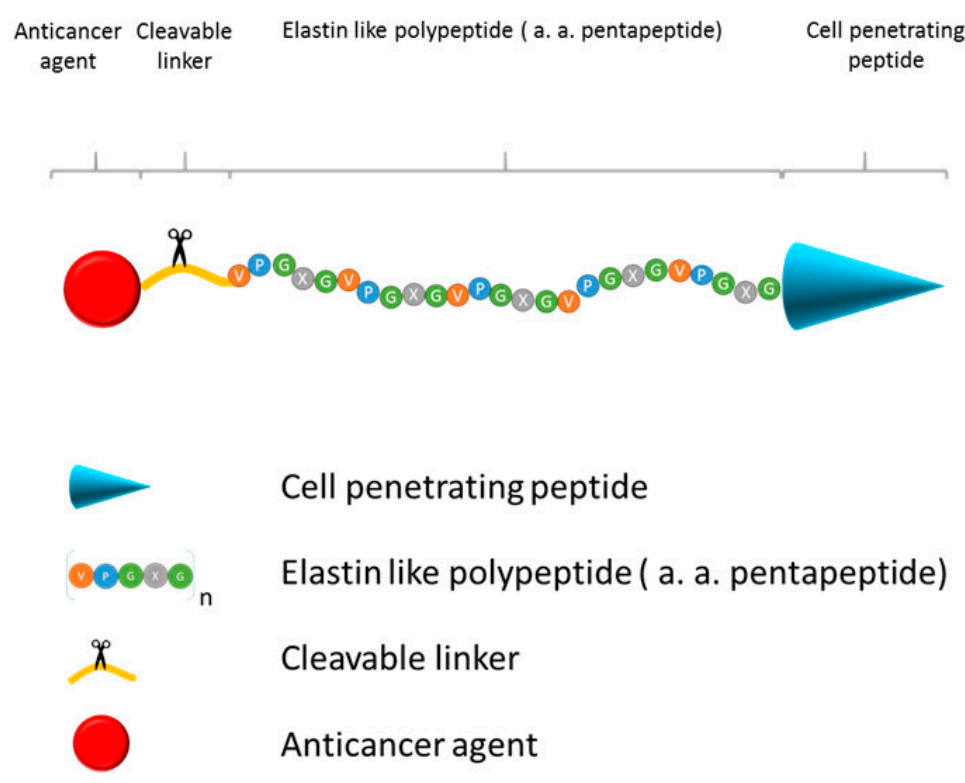

Figure 10. Schematic presentation of Elastin like polypeptide (ELP) polymeric prodrug. Construct consists of (1) Cell penetrating peptide, mediating uptake of ELP in tissues and cancer cells; (2) ELP, thermally responsive biopolymer which can be actively targeted to tumor tissue by external application of mild heat; (3) cleavable linker, releases anticancer agent in tumor tissues and cancer cells, and (4) anticancer agent.

\subsubsection{Silk-Elastin Like Polypeptides}

A silk-like polypeptide is a genetically engineered polypeptide that is composed of (Gly-AlaGly-Ala-Gly-Ser) tandem repeats. For years, silk-like polymers have posed a challenge in that a very low level of their expression is reported when they are derived from the commonly used E. coli host [99]. Utilizing substitute hosts that have higher expression levels such as yeast, insect cells, or transgenic plants may result in higher protein yield. However, these alternative routes may negatively affect protein purity. As a result, silk-like polypeptides are not as broadly used as elastin-like polypeptides, whose solubility facilitates numerous paths to more efficient drug delivery.

To make the silk-like polypeptide more suitable for drug delivery, it is often combined with an ELP. Xiao-Xia et al. [100] constructed a series of silk-elastin like polymers (SELP) which have the capacity to form micellar nanoparticles upon incubation with doxorubicin using heat as a stimuli. These nanoparticles effectively inhibited proliferation of HeLa cell lines, demonstrating the potential use of drug-loaded SELP for cancer treatment.

\section{Polymer Drug Conjugates in Clinical Trials}

Despite the variety of novel drug targets and sophisticated chemistries available, to the best of our knowledge there have been no FDA-approved small molecule prodrugs based on polymer for cancer treatment yet, while only a limited number of polymer-drug conjugates has been placed in the stage of clinical trials. Currently less than 10 polymer-drug conjugates are on-going in clinical trials (Table 2).

\subsection{Dextran Conjugates}

Through GuaDex technology, a DexTech patented technology platform, dextran is modified and used as the backbone for construction of new candidate medications. OsteoDex and Somadex, which are made from alendronate and somatostatin by DexTech, are being tested in clinical trials to treat castration resistant prostate cancer (CRPC) [78]. 


\subsection{HPMA Conjugates}

FCE28068, a conjugate of doxorubicin with HPMA copolymer via GFLG linker, showed 5-fold decreased toxicity compared to doxorubicin in a phase I study [101]. In a phase II trial with breast, non-small cell lung, and colorectal cancer patients, FCE28068 had a total of 6 breast and NSCL cancer patients showing partial responses out of 62 patients without severe toxicities [101]. No subsequent reports have been released.

\subsection{PEG Conjugates}

\subsubsection{EZN-2208/BEL-0222}

SN38 (10-hydroxy-7-ethyl-camptothecin) is a potent topoisomerase I inhibitor and the active moiety of irinotecan (CPT-11). PEG-SN38 is thus a PEGylated conjugate of SN38 for better solubility and an improved pharmacokinetic profile [102]. In addition, the ester bond between the polymer and the drug can be cleaved in PBS, releasing approximately $57 \%$ of the conjugated SN-38 from the micelles [80]. PEG-SN38 has been shown to down-modulate HIF-1 $\alpha$ involved in tumor invasion, migration, angiogenesis and production of vascular endothelial growth factor (VEGF). PEG-SN38 has produced positive responses in clinical trials for metastatic breast cancer and pediatric neuroblastoma and currently is in a phase III trial [103]

\subsubsection{NC-6300}

NC-6300 consists of a PEG polyaspartate block copolymer, an acid-labile hydrazone linker and epirubicin. Preclinical studies using Hep3B/Luc orthotopic hepatic tumor models showed a significant improvement in the survival rate in the group administered NC-6300 at $10 \mathrm{mg} / \mathrm{kg}$ compared with that in the group with EPI at $10 \mathrm{mg} / \mathrm{kg}$ [81]. Currently, Nano Carrier is conducting a phase I clinical trial in Japan [21].

\section{Conclusions and Future Direction}

Poor solubility and selectivity of anti-cancer drugs limits efficacy of the current cancer treatments. Conjugating drugs to polymers improves drug solubility, pharmacokinetic and pharmacodynamics, as well as increasing their tumor specificity. Thus, polymers provide a remarkable tool through which to increase the therapeutic efficacy of drugs. Despite decades of significant progress in polymer-based drug delivery, the number of FDA approved polymer-based drugs remains very small.

Major objectives in designing new polymer based prodrugs include: (1) improving solubility of the drugs in aqueous solution or lipids; (2) conjugating the drug to the polymer without decreasing its potency; (3) binding the drug tightly to a polymer in circulation, but releasing it inside the tumor cells; and (4) ensuring that the polymer is non-immunogenic, biodegradable and can be cost-effectively produced in sufficient quantities. Successfully addressing these challenges will yield novel and efficient polymer-based prodrugs and establish their significant role in current cancer therapy.

Acknowledgements: We would like to express our great appreciation to Jo Anne Fordham of the UMMC Center for Bioethics and Medical Humanities for editorial support. We would further like to acknowledge Rebecca Mackey for helpful discussion.

Author Contributions: Sonja Dragojevic wrote sections Natural polymers, Genetically engineered polymers, and created associated figures, as well as figure in the Introduction. Jung Su Ryu wrote sections Synthetic polymers, Polymer Drug Conjugates in Clinical Trials, and created associated figures and tables. Drazen Raucher coordinated writing of all sections and he wrote Introduction and Conclusions and Future Direction sections. All authors were involved in the editing process.

Conflicts of Interest: The authors declare no conflict of interest. 


\section{References}

1. Williams, H.D.; Trevaskis, N.L.; Charman, S.A.; Shanker, R.M.; Charman, W.N.; Pouton, C.W.; Porter, C.J. Strategies to address low drug solubility in discovery and development. Pharmacol. Rev. 2013, 65, 315-499.

2. Bikiaris, D.N. Solid dispersions, part II: New strategies in manufacturing methods for dissolution rate enhancement of poorly water-soluble drugs. Expert Opin. Drug Deliv. 2011, 8, 1663-1680.

3. Sheth, P.; Sandhu, H.; Singhal, D.; Malick, W.; Shah, N.; Serpil Kislalioglu, M. Nanoparticles in the pharmaceutical industry and the use of supercritical fluid technologies for nanoparticle production. Curr. Drug Deliv. 2012, 9, 269-284.

4. Cooper, E.R. Nanoparticles: A personal experience for formulating poorly water soluble drugs. J. Control. Release 2010, 141, 300-302.

5. Jouyban, A. Review of the cosolvency models for predicting solubility of drugs in water-cosolvent mixtures. J. Pharm. Pharm. Sci. 2008, 11, 32-58.

6. Venkatesh, S.; Li, J.; Xu, Y.; Vishnuvajjala, R.; Anderson, B.D. Intrinsic solubility estimation and pH-solubility behavior of cosalane (NSC 658586), an extremely hydrophobic diprotic acid. Pharm. Res. 1996, 13, 1453-1459.

7. Adams, J.D.; Flora, K.; Goldspiel, B.; Wilson, J.; Arbuck, S.; Finley, R. Taxol: A history of pharmaceutical development and current pharmaceutical concerns. J. Natl. Cancer Inst. Monogr. 1992, 141-147

8. Arbuck, S.G.; Christian, M.; Fisherman, J.; Cazenave, L.; Sarosy, G.; Suffness, M.; Adams, J.; Canetta, R.; Cole, K.; Friedman, M. Clinical development of Taxol. J. Natl. Cancer Inst. Monogr. 1992, 11-24.

9. Dorr, R.T. Pharmacology and toxicology of Cremophor EL diluent. Ann. Pharmacother. 1994, 28, S11-S14.

10. Duncan, R.; Vicent, M.J. Polymer therapeutics-prospects for 21st century: The end of the beginning. Adv. Drug Deliv. Rev. 2013, 65, 60-70.

11. Giang, I.; Boland, E.L.; Poon, G.M. Prodrug applications for targeted cancer therapy. AAPS J. 2014, 16, 899-913.

12. Maeda, H. Macromolecular therapeutics in cancer treatment: The EPR effect and beyond. J. Control. Release 2012, 164, 138-144.

13. John, J.V.; Johnson, R.P.; Heo, M.S.; Moon, B.K.; Byeon, S.J.; Kim, I. Polymer-Block-Polypeptides and Polymer-Conjugated Hybrid Materials as Stimuli-Responsive Nanocarriers for Biomedical Applications. J. Biomed. Nanotechnol. 2015, 11, 1-39.

14. Taghizadeh, B.; Taranejoo, S.; Monemian, S.A.; Salehi Moghaddam, Z.; Daliri, K.; Derakhshankhah, H.; Derakhshani, Z. Classification of stimuli-responsive polymers as anticancer drug delivery systems. Drug Deliv. 2015, 22, 145-155.

15. Ryu, J.S.; Raucher, D. Elastin-like polypeptide for improved drug delivery for anticancer therapy: Preclinical studies and future applications. Expert Opin. Drug Deliv. 2015, 12, 653-667.

16. Pan, H.; Sima, M.; Yang, J.; Kopeček, J. Synthesis of Long-Circulating, Backbone Degradable HPMA Copolymer-Doxorubicin Conjugates and Evaluation of Molecular-Weight-Dependent Antitumor Efficacy. Macromol. Biosci. 2013, 13, 155-160.

17. Duncan, R.; Kopeček, J. Soluble synthetic polymers as potential drug carriers. In Polymers in Medicine; Springer: Berlin, Heidelberg, Germany; 1984; Volume 57, pp. 51-101.

18. Jang, H.-J.; Shin, C.Y.; Kim, K.-B. Safety Evaluation of Polyethylene Glycol (PEG) Compounds for Cosmetic Use. Toxicol. Res. 2015, 31, 105-136.

19. Pang, X.; Du, H.L.; Zhang, H.Q.; Zhai, Y.J.; Zhai, G.X. Polymer-drug conjugates: Present state of play and future perspectives. Drug Discov. Today 2013, 18, 1316-1322.

20. Rohini, N.A.; Joseph, A.; Mukerji, A. Polymeric prodrugs: Recent achievements and general strategies. J. Antivir. Antiretrovir. 2013, doi:10.4172/jaa.S15-007.

21. Alekseenko, I.V.; Snezhkov, E.V.; Chernov, I.P.; Pleshkan, V.V.; Potapov, V.K.; Sass, A.V.; Monastyrskaya, G.S.; Kopantzev, E.P.; Vinogradova, T.V.; Khramtsov, Y.V.; et al. Therapeutic properties of a vector carrying the HSV thymidine kinase and GM-CSF genes and delivered as a complex with a cationic copolymer. J. Transl. Med. 2015, 13, 78, doi:10.1186/s12967-015-0433-0.

22. Huo, M.; Zhu, Q.; Wu, Q.; Yin, T.; Wang, L.; Yin, L.; Zhou, J. Somatostatin receptor-mediated specific delivery of paclitaxel prodrugs for efficient cancer therapy. J. Pharm. Sci. 2015, 104, 2018-2028.

23. Huang, P.; Wang, W.; Zhou, J.; Zhao, F.; Zhang, Y.; Liu, J.; Liu, J.; Dong, A.; Kong, D.; Zhang, J. Amphiphilic polyelectrolyte/prodrug nanoparticles constructed by synergetic electrostatic and hydrophobic interactions with cooperative $\mathrm{pH}$-sensitivity for controlled doxorubicin delivery. ACS Appl. Mater. Interfaces 2015, 7, 6340-6350. 
24. Stefan, N.; Zimmermann, M.; Simon, M.; Zangemeister-Wittke, U.; Pluckthun, A. Novel prodrug-like fusion toxin with protease-sensitive bioorthogonal PEGylation for tumor targeting. Bioconjug. Chem. 2014, 25, 2144-2156.

25. Lv, S.; Tang, Z.; Zhang, D.; Song, W.; Li, M.; Lin, J.; Liu, H.; Chen, X. Well-defined polymer-drug conjugate engineered with redox and $\mathrm{pH}$-sensitive release mechanism for efficient delivery of paclitaxel. J. Control. Release 2014, 194, 220-227.

26. Bao, Y.; Guo, Y.; Zhuang, X.; Li, D.; Cheng, B.; Tan, S.; Zhang, Z. D-alpha-tocopherol polyethylene glycol succinate-based redox-sensitive paclitaxel prodrug for overcoming multidrug resistance in cancer cells. Mol. Pharm. 2014, 11, 3196-3209.

27. Wang, Y.; Wang, H.; Chen, Y.; Liu, X.; Jin, Q.; Ji, J. pH and hydrogen peroxide dual responsive supramolecular prodrug system for controlled release of bioactive molecules. Colloids Surf. B Biointerfaces 2014, 121, 189-195.

28. Nam, J.P.; Park, J.K.; Son, D.H.; Kim, T.H.; Park, S.J.; Park, S.C.; Choi, C.; Jang, M.K.; Nah, J.W. Evaluation of polyethylene glycol-conjugated novel polymeric anti-tumor drug for cancer therapy. Colloids Surf. B Biointerfaces 2014, 120, 168-175.

29. Daman, Z.; Ostad, S.; Amini, M.; Gilani, K. Preparation, optimization and in vitro characterization of stearoyl-gemcitabine polymeric micelles: A comparison with its self-assembled nanoparticles. Int. J. Pharm. 2014, 468, 142-151.

30. Wu, X.; Sun, X.; Guo, Z.; Tang, J.; Shen, Y.; James, T.D.; Tian, H.; Zhu, W. In vivo and in situ tracking cancer chemotherapy by highly photostable NIR fluorescent theranostic prodrug. J. Am. Chem. Soc. 2014, 136, 3579-3588.

31. Meng, H.; Xu, K.; Xu, Y.; Luo, P.; Du, F.; Huang, J.; Lu, W.; Yu, J.; Liu, S.; Muir, B. Nanocapsules based on mPEGylated artesunate prodrug and its cytotoxicity. Colloids Surf. B 2014, 115, 164-169.

32. Xu, Z.; Wang, D.; Xu, S.; Liu, X.; Zhang, X.; Zhang, H. Preparation of a camptothecin prodrug with glutathione-responsive disulfide linker for anticancer drug delivery. Chem. Asian J. 2014, 9, 199-205.

33. Henne, W.A.; Kularatne, S.A.; Hakenjos, J.; Carron, J.D.; Henne, K.L. Synthesis and activity of a folate targeted monodisperse PEG camptothecin conjugate. Bioorg. Med. Chem. Lett. 2013, 23, 5810-5813.

34. Zhu, L.; Wang, T.; Perche, F.; Taigind, A.; Torchilin, V.P. Enhanced anticancer activity of nanopreparation containing an MMP2-sensitive PEG-drug conjugate and cell-penetrating moiety. Proc. Natl. Acad. Sci. USA 2013, 110, 17047-17052.

35. Wei, J.; Wang, H.; Zhu, M.; Ding, D.; Li, D.; Yin, Z.; Wang, L.; Yang, Z. Janus nanogels of PEGylated Taxol and PLGA-PEG-PLGA copolymer for cancer therapy. Nanoscale 2013, 5, 9902-9907.

36. Jiang, T.; Li, Y.M.; Lv, Y.; Cheng, Y.J.; He, F.; Zhuo, R.X. Amphiphilic polycarbonate conjugates of doxorubicin with pH-sensitive hydrazone linker for controlled release. Colloids Surf. B 2013, 111, 542-548.

37. Talelli, M.; Morita, K.; Rijcken, C.J.; Aben, R.W.; Lammers, T.; Scheeren, H.W.; van Nostrum, C.F.; Storm, G.; Hennink, W.E. Synthesis and characterization of biodegradable and thermosensitive polymeric micelles with covalently bound doxorubicin-glucuronide prodrug via click chemistry. Bioconjug. Chem. 2011, 22, 2519-2530.

38. Shamay, Y.; Raviv, L.; Golan, M.; Voronov, E.; Apte, R.N.; David, A. Inhibition of primary and metastatic tumors in mice by E-selectin-targeted polymer-drug conjugates. J. Control. Release 2015, 217, 102-112.

39. Zhong, J.; Li, L.; Zhu, X.; Guan, S.; Yang, Q.; Zhou, Z.; Zhang, Z.; Huang, Y. A smart polymeric platform for multistage nucleus-targeted anticancer drug delivery. Biomaterials 2015, 65, 43-55.

40. Peng, Z.H.; Kopecek, J. Enhancing Accumulation and Penetration of HPMA Copolymer-Doxorubicin Conjugates in 2D and 3D Prostate Cancer Cells via iRGD Conjugation with an MMP-2 Cleavable Spacer. J. Am. Chem. Soc. 2015, 137, 6726-6729.

41. Yang, Q.; Yang, Y.; Li, L.; Sun, W.; Zhu, X.; Huang, Y. Polymeric nanomedicine for tumor-targeted combination therapy to elicit synergistic genotoxicity against prostate cancer. ACS Appl. Mater. Interfaces 2015, 7, 6661-6673.

42. Chytil, P.; Koziolova, E.; Janouskova, O.; Kostka, L.; Ulbrich, K.; Etrych, T. Synthesis and Properties of Star HPMA Copolymer Nanocarriers Synthesised by RAFT Polymerisation Designed for Selective Anticancer Drug Delivery and Imaging. Macromol. Biosci. 2015, 15, 839-850.

43. Nakamura, H.; Koziolova, E.; Etrych, T.; Chytil, P.; Fang, J.; Ulbrich, K.; Maeda, H. Comparison between linear and star-like HPMA conjugated pirarubicin (THP) in pharmacokinetics and antitumor activity in tumor bearing mice. Eur. J. Pharm. Biopharm. 2015, 90, 90-96. 
44. Nakamura, H.; Etrych, T.; Chytil, P.; Ohkubo, M.; Fang, J.; Ulbrich, K.; Maeda, H. Two step mechanisms of tumor selective delivery of $\mathrm{N}$-(2-hydroxypropyl)methacrylamide copolymer conjugated with pirarubicin via an acid-cleavable linkage. J. Control. Release 2014, 174, 81-87.

45. Sedlacek, O.; Kucka, J.; Mattova, J.; Parizek, M.; Studenovsky, M.; Zadinova, M.; Pouckova, P.; Hruby, M. Multistage-targeted $\mathrm{pH}$-responsive polymer conjugate of Auger electron emitter: optimized design and in vivo activity. Eur. J. Pharm. Sci. 2014, 63, 216-225.

46. Zhang, R.; Yang, J.; Sima, M.; Zhou, Y.; Kopecek, J. Sequential combination therapy of ovarian cancer with degradable $N$-(2-hydroxypropyl)methacrylamide copolymer paclitaxel and gemcitabine conjugates. Proc. Natl. Acad. Sci. USA 2014, 111, 12181-12186.

47. Mohanraj, S.; Murugan, D.; Rengarajan, A.; Rajiv, S. Anticancer activity of starch/poly[N-(2-hydroxypropyl) methacrylamide]: Biomaterial film to treat skin cancer. Int. J. Biol. Macromol. 2014, 70, 116-123.

48. Wu, Y.; Ji, J.; Yang, R.; Zhang, X.; Li, Y.; Pu, Y.; Li, X. Galactosylated 2-hydroxypropyl methacrylamide-s3-guanidinopropyl methacrylamide copolymer as a small hairpin RNA carrier for inhibiting human telomerase reverse transcriptase expression. J. Gene Med. 2014, 16, 109-121.

49. Buckway, B.; Frazier, N.; Gormley, A.J.; Ray, A.; Ghandehari, H. Gold nanorod-mediated hyperthermia enhances the efficacy of HPMA copolymer-90Y conjugates in treatment of prostate tumors. Nucl. Med. Biol. 2014, 41, 282-289.

50. Larson, N.; Yang, J.; Ray, A.; Cheney, D.L.; Ghandehari, H.; Kopecek, J. Biodegradable multiblock poly(N-2-hydroxypropyl)methacrylamide gemcitabine and paclitaxel conjugates for ovarian cancer cell combination treatment. Int. J. Pharm. 2013, 454, 435-443.

51. Nakamura, H.; Liao, L.; Hitaka, Y.; Tsukigawa, K.; Subr, V.; Fang, J.; Ulbrich, K.; Maeda, H. Micelles of zinc protoporphyrin conjugated to $\mathrm{N}$-(2-hydroxypropyl)methacrylamide (HPMA) copolymer for imaging and light-induced antitumor effects in vivo. J. Control. Release 2013, 165, 191-198.

52. Kayat, J.; Mehra, N.K.; Gajbhiye, V.; Jain, N.K. Drug targeting to arthritic region via folic acid appended surface-engineered multi-walled carbon nanotubes. J. Drug Targets 2015, 1-10.

53. Khare, V.; Kour, S.; Alam, N.; Dubey, R.D.; Saneja, A.; Koul, M.; Gupta, A.P.; Singh, D.; Singh, S.K.; Saxena, A.K.; et al. Synthesis, characterization and mechanistic-insight into the anti-proliferative potential of PLGA-gemcitabine conjugate. Int. J. Pharm. 2014, 470, 51-62.

54. Van, S.; Das, S. K.; Wang, X.; Feng, Z.; Jin, Y.; Hou, Z.; Chen, F.; Pham, A.; Jiang, N.; Howell, S. B. Synthesis, characterization, and biological evaluation of poly (L- $\gamma$-glutamyl-glutamine)-paclitaxel nanoconjugate. Int.J. Nanomed. 2010, 5, 825-837.

55. Yang, D.; Liu, X.; Jiang, X.; Liu, Y.; Ying, W.; Wang, H.; Bai, H.; Taylor, W.D.; Wang, Y.; Clamme, J.-P. Effect of molecular weight of PGG-paclitaxel conjugates on in vitro and in vivo efficacy. J. Control. Release 2012, 161, 124-131.

56. Cai, L.; Xu, G.; Shi, C.; Guo, D.; Wang, X.; Luo, J. Telodendrimer nanocarrier for co-delivery of paclitaxel and cisplatin: A synergistic combination nanotherapy for ovarian cancer treatment. Biomaterials 2015, 37, 456-468.

57. Xiao, H.; Song, H.; Yang, Q.; Cai, H.; Qi, R.; Yan, L.; Liu, S.; Zheng, Y.; Huang, Y.; Liu, T. A prodrug strategy to deliver cisplatin (IV) and paclitaxel in nanomicelles to improve efficacy and tolerance. Biomaterials 2012, 33, 6507-6519.

58. Duncan, R.; Vicent, M.J. Do HPMA copolymer conjugates have a future as clinically useful nanomedicines? A critical overview of current status and future opportunities. Adv. Drug Deliv. Rev. 2010, 62, 272-282.

59. Larson, N.; Greish, K.; Bauer, H.; Maeda, H.; Ghandehari, H. Synthesis and evaluation of poly(styrene-comaleic acid) micellar nanocarriers for the delivery of tanespimycin. Int. J. Pharm. 2011, 420, 111-117.

60. Mu, Y.; Kamada, H.; Kaneda, Y.; Yamamoto, Y.; Kodaira, H.; Tsunoda, S.; Tsutsumi, Y.; Maeda, M.; Kawasaki, K.; Nomizu, M.; et al. YBioconjugation of laminin peptide YIGSR with poly(styrene co-maleic acid) increases its antimetastatic effect on lung metastasis of B16-BL6 melanoma cells. Biochem. Biophys. Res. Commun. 1999, 255, 75-79.

61. Maeda, H. SMANCS and polymer-conjugated macromolecular drugs: Advantages in cancer chemotherapy. Adv. Drug Deliv. Rev. 2001, 46, 169-185.

62. Maeda, H.; Ueda, M.; Morinaga, T.; Matsumoto, T. Conjugation of poly(styrene-co-maleic acid) derivatives to the antitumor protein neocarzinostatin: Pronounced improvements in pharmacological properties. J. Med. Chem. 1985, 28, 455-461. 
63. Fang, J.; Tsukigawa, K.; Liao, L.; Yin, H.; Eguchi, K.; Maeda, H. Styrene-maleic acid-copolymer conjugated zinc protoporphyrin as a candidate drug for tumor-targeted therapy and imaging. J. Drug Target. 2015, 1-9.

64. Bae, H.H.; Cho, M.Y.; Hong, J.H.; Poo, H.; Sung, M.H.; Lim, Y.T. Bio-derived poly(gamma-glutamic acid) nanogels as controlled anticancer drug delivery carriers. J. Microbiol. Biotechnol. 2012, 22, 1782-1789.

65. Seth, A.; Heo, M.B.; Lim, Y.T. Poly (gamma-glutamic acid) based combination of water-insoluble paclitaxel and TLR7 agonist for chemo-immunotherapy. Biomaterials 2014, 35, 7992-8001.

66. Makadia, H.K.; Siegel, S.J. Poly Lactic-co-Glycolic Acid (PLGA) as Biodegradable Controlled Drug Delivery Carrier. Polymers 2011, 3, 1377-1397.

67. Emami, J.; Rezazadeh, M.; Hasanzadeh, F.; Sadeghi, H.; Mostafavi, A.; Minaiyan, M.; Rostami, M.; Davies, N. Development and in vitro/in vivo evaluation of a novel targeted polymeric micelle for delivery of paclitaxel. Int. J. Biol. Macromol. 2015, 80, 29-40.

68. Cheng, C.J.; Bahal, R.; Babar, I.A.; Pincus, Z.; Barrera, F.; Liu, C.; Svoronos, A.; Braddock, D.T.; Glazer, P.M.; Engelman, D.M.; et al. MicroRNA silencing for cancer therapy targeted to the tumour microenvironment. Nature 2015, 518, 107-110.

69. Gorska, A.; Swiatkowska, A.; Dutkiewicz, M.; Ciesiolka, J. Modulation of p53 expression using antisense oligonucleotides complementary to the 5 '-terminal region of p53 mRNA in vitro and in the living cells. PLOS ONE 2013, 8, e78863.

70. Xiang, D.; Shigdar, S.; Qiao, G.; Wang, T.; Kouzani, A.Z.; Zhou, S.F.; Kong, L.; Li, Y.; Pu, C.; Duan, W. Nucleic acid aptamer-guided cancer therapeutics and diagnostics: The next generation of cancer medicine. Theranostics 2015, 5, 23-42.

71. Qi, L.; Xu, Z. In vivo antitumor activity of chitosan nanoparticles. Bioorg. Med. Chem. Lett. 2006, 16, 4243-4245.

72. Qi, L.; Xu, Z.; Chen, M. In vitro and in vivo suppression of hepatocellular carcinoma growth by chitosan nanoparticles. Eur. J. Cancer 2007, 43, 184-193.

73. Nogueira, D.R.; Scheeren, L.E.; Macedo, L.B.; Marcolino, A.I.; Pilar Vinardell, M.; Mitjans, M.; Rosa Infante, M.; Farooqi, A.A.; Rolim, C.M. Inclusion of a pH-responsive amino acid-based amphiphile in methotrexate-loaded chitosan nanoparticles as a delivery strategy in cancer therapy. Amino Acids 2015, in press.

74. Banerjee, T.; Mitra, S.; Kumar Singh, A.; Kumar Sharma, R.; Maitra, A. Preparation, characterization and biodistribution of ultrafine chitosan nanoparticles. Int. J. Pharm. 2002, 243, 93-105.

75. Rubino, F.M. Separation methods for methotrexate, its structural analogues and metabolites. J. Chromatogr. $B$ 2001, 764, 217-254.

76. Nam, J.P.; Lee, K.J.; Choi, J.W.; Yun, C.O.; Nah, J.W. Targeting delivery of tocopherol and doxorubicin grafted-chitosan polymeric micelles for cancer therapy: In vitro and in vivo evaluation. Colloids Surf. B 2015, 133, 254-262.

77. Cheung, R.Y.; Rauth, A.M.; Ronaldson, P.T.; Bendayan, R.; Wu, X.Y. In vitro toxicity to breast cancer cells of microsphere-delivered mitomycin $\mathrm{C}$ and its combination with doxorubicin. Eur. J. Pharm. Biopharm. 2006, 62, 321-331.

78. DexTch Medical. Available online:http://dextechmedical.com (accessed on 30 November 2015)

79. ClinicalTrials.gov. Available online:http://clinicaltrials.gov (NCT01595087) (accessed on 30 November 2015).

80. Cabral, H.; Kataoka, K. Progress of drug-loaded polymeric micelles into clinical studies. J. Control. Release 2014, 190, 465-476.

81. Matsumura, Y. The drug discovery by nanomedicine and its clinical experience. Jpn. J. Clin. Oncol. 2014, $44,515-525$.

82. CTI BioPharma. Available online:http://www.ctibiopharma.com (accessed on 30 November 2015).

83. Langer, C.J.; O’Byrne, K.J.; Socinski, M.A.; Mikhailov, S.M.; Lesniewski-Kmak, K.; Smakal, M.; Ciuleanu, T.E.; Orlov, S.V.; Dediu, M.; Heigener, D.; et al. Phase III trial comparing paclitaxel poliglumex (CT-2103, PPX) in combination with carboplatin versus standard paclitaxel and carboplatin in the treatment of PS 2 patients with chemotherapy-naive advanced non-small cell lung cancer. J. Thorac. Oncol. 2008, 3, 623-630.

84. Cerlulean Available online:http://www,ceruleanrx.com (accessed on 30 November 2015).

85. Lee, K.D.; Choi, S.H.; Kim da, H.; Lee, H.Y.; Choi, K.C. Self-organized nanoparticles based on chitosan-folic acid and dextran succinate-doxorubicin conjugates for drug targeting. Arch. Pharm. Res. 2014, 37, 1546-1553.

86. Ganeshkumar, M.; Ponrasu, T.; Raja, M.D.; Subamekala, M.K.; Suguna, L. Green synthesis of pullulan stabilized gold nanoparticles for cancer targeted drug delivery. Spectrochim. Acta A Mol. Biomol. Spectrosc. 2014, 130, 64-71. 
87. Scomparin, A.; Salmaso, S.; Bersani, S.; Satchi-Fainaro, R.; Caliceti, P. Novel folated and non-folated pullulan bioconjugates for anticancer drug delivery. Eur. J. Pharm. Sci. 2011, 42, 547-558.

88. Malet-Martino, M.; Martino, R. Clinical studies of three oral prodrugs of 5-fluorouracil (capecitabine, UFT, S-1): A review. Oncologist 2002, 7, 288-323.

89. Zhang, H.; Li, F.; Yi, J.; Gu, C.; Fan, L.; Qiao, Y.; Tao, Y.; Cheng, C.; Wu, H. Folate-decorated maleilated pullulan-doxorubicin conjugate for active tumor-targeted drug delivery. Eur. J. Pharm. Sci. 2011, 42, 517-526.

90. Moktan, S.; Ryppa, C.; Kratz, F.; Raucher, D. A thermally responsive biopolymer conjugated to an acid-sensitive derivative of paclitaxel stabilizes microtubules, arrests cell cycle, and induces apoptosis. Investig. New Drugs 2012, 30, 236-248.

91. Mikecin, A.M.; Walker, L.R.; Kuna, M.; Raucher, D., Thermally targeted p21 peptide enhances bortezomib cytotoxicity in androgen-independent prostate cancer cell lines. Anticancer Drugs 2014, 25, 189-199.

92. Moktan, S.; Perkins, E.; Kratz, F.; Raucher, D. Thermal targeting of an acid-sensitive doxorubicin conjugate of elastin-like polypeptide enhances the therapeutic efficacy compared with the parent compound in vivo. Mol. Cancer Ther. 2012, 11, 1547-1556.

93. Massodi, I.; Bidwell, G.L., 3rd; Davis, A.; Tausend, A.; Credit, K.; Flessner, M.; Raucher, D. Inhibition of ovarian cancer cell metastasis by a fusion polypeptide Tat-ELP. Clin. Exp. Metastasis 2009, 26, 251-260.

94. Bidwell, G.L., 3rd; Perkins, E.; Hughes, J.; Khan, M.; James, J.R.; Raucher, D. Thermally targeted delivery of a c-Myc inhibitory polypeptide inhibits tumor progression and extends survival in a rat glioma model. PLOS ONE 2013, 8, e55104.

95. Bidwell, G.L., 3rd; George, E.M. Maternally sequestered therapeutic polypeptides-A new approach for the management of preeclampsia. Front. Pharmacol. 2014, 5, 201, doi:10.3389/fphar.2014.00201.

96. Amruthwar, S.S.; Janorkar, A.V. In vitro evaluation of elastin-like polypeptide-collagen composite scaffold for bone tissue engineering. Dent. Mater.2013, 29, 211-220.

97. Bidwell, G.L., 3rd; Davis, A.N.; Fokt, I.; Priebe, W.; Raucher, D. A thermally targeted elastin-like polypeptide-doxorubicin conjugate overcomes drug resistance. Investig. New Drugs 2007, 25, 313-326.

98. Andrew MacKay, J.; Chen, M.; McDaniel, J.R.; Liu, W.; Simnick, A.J.; Chilkoti, A. Self-assembling chimeric polypeptide-doxorubicin conjugate nanoparticles that abolish tumours after a single injection. Nat. Mater. 2009, 8, 993-999.

99. Chow, D.; Nunalee, M.L.; Lim, D.W.; Simnick, A.J.; Chilkoti, A. Peptide-based Biopolymers in Biomedicine and Biotechnology. Mater. Sci. Eng. R Rep. 2008, 62, 125-155.

100. Xia, X.X.; Wang, M.; Lin, Y.; Xu, Q.; Kaplan, D.L. Hydrophobic drug-triggered self-assembly of nanoparticles from silk-elastin-like protein polymers for drug delivery. Biomacromolecules 2014, 15, 908-914.

101. Seymour, L.W.; Ferry, D.R.; Kerr, D.J.; Rea, D.; Whitlock, M.; Poyner, R.; Boivin, C.; Hesslewood, S.; Twelves, C.; Blackie, R.; et al. Phase II studies of polymer-doxorubicin (PK1, FCE28068) in the treatment of breast, lung and colorectal cancer. Int. J. Oncol. 2009, 34, 1629-1636.

102. Patnaik, A.; Papadopoulos, K.P.; Tolcher, A.W.; Beeram, M.; Urien, S.; Schaaf, L.J.; Tahiri, S.; Bekaii-Saab, T.; Lokiec, F.M.; Rezai, K.; et al. Phase I dose-escalation study of EZN-2208 (PEG-SN38), a novel conjugate of poly(ethylene) glycol and SN38, administered weekly in patients with advanced cancer. Cancer Chemother. Pharmacol. 2013, 71, 1499-1506.

103. BelRose Pharma. Available online:http://belrosepharma.com (accessed on 30 November 2015).

(C) 2015 by the authors; licensee MDPI, Basel, Switzerland. This article is an open access article distributed under the terms and conditions of the Creative Commons by Attribution (CC-BY) license (http://creativecommons.org/licenses/by/4.0/). 